CERN-EP-2002-094

20 December 2002

\title{
A Measurement of Semileptonic B Decays to Narrow Orbitally-Excited Charm Mesons
}

\author{
The OPAL Collaboration
}

\begin{abstract}
The decay chain $\mathrm{b} \rightarrow \overline{\mathrm{B}} \rightarrow \mathrm{D}^{* * 0} \ell^{-} \bar{\nu} X, \mathrm{D}^{* * 0} \rightarrow \mathrm{D}^{*+} \pi^{-}, \mathrm{D}^{*+} \rightarrow \mathrm{D}^{0} \pi^{+}, \mathrm{D}^{0} \rightarrow(\mathrm{K} \pi$ or $\mathrm{K} 3 \pi)$ is identified in a sample of 3.9 million hadronic $\mathrm{Z}$ decays collected with the OPAL detector at LEP. The branching ratio $\mathrm{BR}(\mathrm{b} \rightarrow \overline{\mathrm{B}}) \times \mathrm{BR}\left(\overline{\mathrm{B}} \rightarrow \mathrm{D}_{1}^{0} \ell^{-} \bar{\nu} X\right) \times \mathrm{BR}\left(\mathrm{D}_{1}^{0} \rightarrow \mathrm{D}^{*+} \pi^{-}\right)$is measured to be $(2.64 \pm 0.79$ (stat) \pm 0.39 (syst) $) \times 10^{-3}$ for the $J^{P}=1^{+}\left(\mathrm{D}_{1}^{0}\right)$ state. For decays into the $J^{P}=2^{+}\left(\mathrm{D}_{2}^{* 0}\right)$ state, an upper limit of $1.4 \times 10^{-3}$ is placed on the branching ratio at the $95 \%$ confidence level.
\end{abstract}

To be submitted to European Journal of Physics C 


\section{The OPAL Collaboration}

G. Abbiendi ${ }^{2}$, C. Ainsley ${ }^{5}$, P.F. Åkesson ${ }^{3}$, G. Alexander ${ }^{22}$, J. Allison ${ }^{16}$, P. Amaral ${ }^{9, a a}$, G. Anagnostou ${ }^{1}$, K.J. Anderson ${ }^{9}$, S. Arcelli ${ }^{2}$, S. Asai ${ }^{23}$, D. Axen ${ }^{27}$, G. Azuelos ${ }^{18, a}$, I. Bailey ${ }^{26}$, E. Barberio ${ }^{8, p}$, R.J. Barlow ${ }^{16}$, R.J. Batley ${ }^{5}$, P. Bechtle ${ }^{25}$, T. Behnke ${ }^{25}$, K.W. Bell ${ }^{20}$, P.J. Bell ${ }^{1}$, G. Bella ${ }^{22}$, A. Bellerive ${ }^{6}$, G. Benelli ${ }^{4}$, S. Bethke ${ }^{32}$, O. Biebel ${ }^{31}$, I.J. Bloodworth ${ }^{1}$, O. Boeriu ${ }^{10}$, P. Bock ${ }^{11}$, D. Bonacorsi ${ }^{2}$, M. Boutemeur ${ }^{31}$, S. Braibant ${ }^{8}$, L. Brigliadori ${ }^{2}$, R.M. Brown ${ }^{20}$, K. Buesser ${ }^{25}$, H.J. Burckhart ${ }^{8}$, S. Campana ${ }^{4}$, R.K. Carnegie ${ }^{6}$, B. Caron $^{28}$, A.A. Carter ${ }^{13}$, J.R. Carter ${ }^{5}$, C.Y. Chang ${ }^{17}$, D.G. Charlton ${ }^{1, b}$, A. Csilling ${ }^{8, g}$, M. Cuffiani ${ }^{2}$, S. Dado ${ }^{21}$, S. Dallison ${ }^{16}$, A.De Roeck ${ }^{8}$, E.A. De Wolf ${ }^{8, s}$, K. $\operatorname{Desch}^{25}$, B. Dienes ${ }^{30}$, M.Donkers ${ }^{6}$, J. Dubbert $^{31}$, E. Duchovni ${ }^{24}$, G. Duckeck ${ }^{31}$, I.P. Duerdoth ${ }^{16}$, E. Elfgren ${ }^{18}$, E. Etzion ${ }^{22}$,

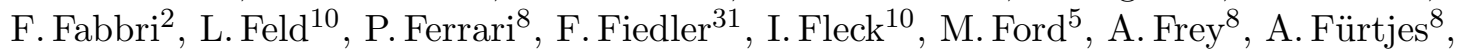
P. Gagnon ${ }^{12}$, J.W. Gary ${ }^{4}$, G. Gaycken ${ }^{25}$, C. Geich-Gimbel ${ }^{3}$, G. Giacomelli ${ }^{2}$, P. Giacomelli ${ }^{2}$, M. Giunta ${ }^{4}$, J. Goldberg ${ }^{21}$, E. Gross ${ }^{24}$, J. Grunhaus ${ }^{22}$, M. Gruwé ${ }^{8}$, P.O. Günther ${ }^{3}$, A. Gupta ${ }^{9}$, C. Hajdu ${ }^{29}$, M.Hamann ${ }^{25}$, G.G.Hanson ${ }^{4}$, K.Harder ${ }^{25}$, A.Harel ${ }^{21}$, M.Harin-Dirac ${ }^{4}$, M. Hauschild ${ }^{8}$, J.Hauschildt ${ }^{25}$, C.M.Hawkes ${ }^{1}$, R. Hawkings ${ }^{8}$, R.J. Hemingway ${ }^{6}$, C. Hensel ${ }^{25}$, G. Herten ${ }^{10}$, R.D. Heuer ${ }^{25}$, J.C. Hill ${ }^{5}$, K. Hoffman ${ }^{9}$, R.J.Homer ${ }^{1}$, D. Horváth ${ }^{29, c}$, R. Howard ${ }^{27}$, P. Igo-Kemenes ${ }^{11}$, K. Ishiii ${ }^{23}$, H. Jeremie ${ }^{18}$, P. Jovanovic ${ }^{1}$, T.R. Junk ${ }^{6}$, N. Kanaya ${ }^{26}$, J. Kanzaki $^{23}$, G. Karapetian ${ }^{18}$, D. Karlen ${ }^{6}$, V. Kartvelishvili ${ }^{16}$, K. Kawagoe ${ }^{23}$, T. Kawamoto ${ }^{23}$, R.K. Keeler ${ }^{26}$, R.G. Kellogg ${ }^{17}$, B.W. Kennedy ${ }^{20}$, D.H. Kim ${ }^{19}$, K. Klein ${ }^{11, t}$, A. Klier ${ }^{24}$, S. Kluth ${ }^{32}$, T.Kobayashi ${ }^{23}$, M. Kobel ${ }^{3}$, S. Komamiya ${ }^{23}$, L. Kormos ${ }^{26}$, T.Krämer ${ }^{25}$, T. Kress ${ }^{4}$, P. Krieger ${ }^{6, l}$, J. von Krogh ${ }^{11}$, D. Krop ${ }^{12}$, K. Kruger ${ }^{8}$, T. Kuhl ${ }^{25}$, M. Kupper ${ }^{24}$, G.D. Lafferty ${ }^{16}$,

H. Landsman ${ }^{21}$, D. Lanske ${ }^{14}$, J.G. Layter ${ }^{4}$, A. Leins ${ }^{31}$, D. Lellouch ${ }^{24}$, J. Letts ${ }^{\text {, }}$, L. Levinson ${ }^{24}$, J. Lillich ${ }^{10}$, S.L. Lloyd ${ }^{13}$, F.K. Loebinger ${ }^{16}$, J. Lu ${ }^{27}$, J. Ludwig ${ }^{10}$, A. Macpherson ${ }^{28, i}$, W. Mader ${ }^{3}$,

S. Marcellini ${ }^{2}$, T.E. Marchant ${ }^{16}$, A.J. Martin ${ }^{13}$, J.P. Martin ${ }^{18}$, G. Masetti ${ }^{2}$, T. Mashimo ${ }^{23}$,

P. Mättig ${ }^{m}$, W.J. McDonald ${ }^{28}$, J. McKenna ${ }^{27}$, T.J. McMahon ${ }^{1}$, R.A. McPherson ${ }^{26}$, F. Meijers ${ }^{8}$, P. Mendez-Lorenzo ${ }^{31}$, W. Menges ${ }^{25}$, F.S. Merritt ${ }^{9}$, H. Mes $^{6, a}$, A. Michelini' ${ }^{2}$, S. Mihara ${ }^{23}$, G. Mikenberg ${ }^{24}$, D.J.Miller ${ }^{15}$, S. Moed $^{21}$, W. Mohr ${ }^{10}$, T. Mori ${ }^{23}$, A. Mutter ${ }^{10}$, K. Nagai ${ }^{13}$, I. Nakamura ${ }^{23}$, H.A. Neal ${ }^{33}$, R. Nisius ${ }^{32}$, S.W. O'Neale ${ }^{1}$, A. Oh ${ }^{8}$, A. Okpara ${ }^{11}$, M.J. Oreglia ${ }^{9}$, S. Orito ${ }^{23}$, C. Pahl ${ }^{32}$, G. Pásztor ${ }^{4, g}$, J.R. Pater ${ }^{16}$, G.N. Patrick ${ }^{20}$, J.E. Pilcher ${ }^{9}$, J. Pinfold ${ }^{28}$,

D.E.Plane ${ }^{8}$, B. Poli ${ }^{2}$, J.Polok ${ }^{8}$, O. Pooth ${ }^{14}$, M.Przybycień ${ }^{8, n}$, A. Quadt ${ }^{3}$, K. Rabbertz ${ }^{8, r}$, C. Rembser ${ }^{8}$, P. Renkel ${ }^{24}$, H. Rick ${ }^{4}$, J.M. Roney ${ }^{26}$, S. Rosati $^{3}$, Y. Rozen ${ }^{21}$, K. Runge ${ }^{10}$, K.Sachs ${ }^{6}$, T.Saeki ${ }^{23}$, O.Sahr ${ }^{31}$, E.K.G.Sarkisyan ${ }^{8, j}$, A.D.Schaile ${ }^{31}$, O. Schaile ${ }^{31}$, P. Scharff-Hansen ${ }^{8}$, J. Schieck ${ }^{32}$, T. Schörner-Sadenius ${ }^{8}$, M. Schröder ${ }^{8}$, M. Schumacher ${ }^{3}$, C. Schwick ${ }^{8}$, W.G. Scott ${ }^{20}$, R.Seuster ${ }^{14, f}$, T.G.Shears ${ }^{8, h}$, B.C.Shen ${ }^{4}$, P. Sherwood ${ }^{15}$, G. Siroli ${ }^{2}$, A. Skuja ${ }^{17}$, A.M. Smith ${ }^{8}$, R. Sobie ${ }^{26}$, S.Söldner-Rembold ${ }^{10, d}$, F. Spano ${ }^{9}$, A.Stahl ${ }^{3}$, K. Stephens ${ }^{16}$, D. Strom ${ }^{19}$, R. Ströhmer ${ }^{31}$, S. Tarem ${ }^{21}$, M. Tasevsky ${ }^{8}$, R.J. Taylor ${ }^{15}$,

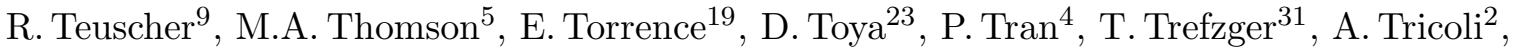
I. Trigger ${ }^{8}$, Z. Trócsányi ${ }^{30, e}$, E. Tsur ${ }^{22}$, M.F. Turner-Watson ${ }^{1}$, I. Ueda ${ }^{23}$, B. Ujvári ${ }^{30, e}$, B. Vachon ${ }^{26}$, C.F. Vollmer ${ }^{31}$, P. Vannerem ${ }^{10}$, M. Verzocchi ${ }^{17}$, H. Voss $^{8, q}$, J. Vossebeld ${ }^{8, h}$,

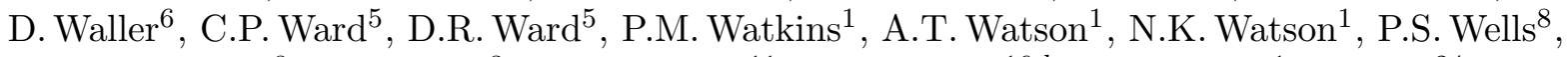
T. Wengler ${ }^{8}$, N. Wermes ${ }^{3}$, D. Wetterling ${ }^{11}$ G.W. Wilson ${ }^{16, k}$, J.A. Wilson ${ }^{1}$, G. Wolf ${ }^{24}$, T.R. Wyatt ${ }^{16}$, S. Yamashita ${ }^{23}$, D. Zer-Zion ${ }^{4}$, L. Zivkovic ${ }^{24}$

\footnotetext{
${ }^{1}$ School of Physics and Astronomy, University of Birmingham, Birmingham B15 2TT, UK

${ }^{2}$ Dipartimento di Fisica dell' Università di Bologna and INFN, I-40126 Bologna, Italy

${ }^{3}$ Physikalisches Institut, Universität Bonn, D-53115 Bonn, Germany

${ }^{4}$ Department of Physics, University of California, Riverside CA 92521, USA
} 
${ }^{5}$ Cavendish Laboratory, Cambridge CB3 0HE, UK

${ }^{6}$ Ottawa-Carleton Institute for Physics, Department of Physics, Carleton University, Ottawa, Ontario K1S 5B6, Canada

${ }^{8}$ CERN, European Organisation for Nuclear Research, CH-1211 Geneva 23, Switzerland

${ }^{9}$ Enrico Fermi Institute and Department of Physics, University of Chicago, Chicago IL 60637, USA

${ }^{10}$ Fakultät für Physik, Albert-Ludwigs-Universität Freiburg, D-79104 Freiburg, Germany

${ }^{11}$ Physikalisches Institut, Universität Heidelberg, D-69120 Heidelberg, Germany

${ }^{12}$ Indiana University, Department of Physics, Bloomington IN 47405, USA

${ }^{13}$ Queen Mary and Westfield College, University of London, London E1 4NS, UK

${ }^{14}$ Technische Hochschule Aachen, III Physikalisches Institut, Sommerfeldstrasse 26-28, D-52056

Aachen, Germany

${ }^{15}$ University College London, London WC1E 6BT, UK

${ }^{16}$ Department of Physics, Schuster Laboratory, The University, Manchester M13 9PL, UK

${ }^{17}$ Department of Physics, University of Maryland, College Park, MD 20742, USA

${ }^{18}$ Laboratoire de Physique Nucléaire, Université de Montréal, Montréal, Québec H3C 3J7, Canada

${ }^{19}$ University of Oregon, Department of Physics, Eugene OR 97403, USA

${ }^{20}$ CLRC Rutherford Appleton Laboratory, Chilton, Didcot, Oxfordshire OX11 0QX, UK

${ }^{21}$ Department of Physics, Technion-Israel Institute of Technology, Haifa 32000, Israel

${ }^{22}$ Department of Physics and Astronomy, Tel Aviv University, Tel Aviv 69978, Israel

${ }^{23}$ International Centre for Elementary Particle Physics and Department of Physics, University of Tokyo, Tokyo 113-0033, and Kobe University, Kobe 657-8501, Japan

${ }^{24}$ Particle Physics Department, Weizmann Institute of Science, Rehovot 76100, Israel

${ }^{25}$ Universität Hamburg/DESY, Institut für Experimentalphysik, Notkestrasse 85, D-22607 Hamburg, Germany

${ }^{26}$ University of Victoria, Department of Physics, P O Box 3055, Victoria BC V8W 3P6, Canada

${ }^{27}$ University of British Columbia, Department of Physics, Vancouver BC V6T 1Z1, Canada

${ }^{28}$ University of Alberta, Department of Physics, Edmonton AB T6G 2J1, Canada

${ }^{29}$ Research Institute for Particle and Nuclear Physics, H-1525 Budapest, P O Box 49, Hungary

${ }^{30}$ Institute of Nuclear Research, H-4001 Debrecen, P O Box 51, Hungary

${ }^{31}$ Ludwig-Maximilians-Universität München, Sektion Physik, Am Coulombwall 1, D-85748 Garching, Germany

${ }^{32}$ Max-Planck-Institute für Physik, Föhringer Ring 6, D-80805 München, Germany

${ }^{33}$ Yale University, Department of Physics, New Haven, CT 06520, USA

${ }^{a}$ and at TRIUMF, Vancouver, Canada V6T $2 \mathrm{~A} 3$

${ }^{b}$ and Royal Society University Research Fellow

${ }^{c}$ and Institute of Nuclear Research, Debrecen, Hungary

$d$ and Heisenberg Fellow

$e^{e}$ and Department of Experimental Physics, Lajos Kossuth University, Debrecen, Hungary

$f$ and MPI München

$g$ and Research Institute for Particle and Nuclear Physics, Budapest, Hungary

$h$ now at University of Liverpool, Dept of Physics, Liverpool L69 3BX, U.K.

${ }^{i}$ and CERN, EP Div, 1211 Geneva 23

${ }^{j}$ now at University of Nijmegen, HEFIN, NL-6525 ED Nijmegen, The Netherlands, on NWO/NATO

Fellowship B 64-29

${ }^{k}$ now at University of Kansas, Dept of Physics and Astronomy, Lawrence, KS 66045, U.S.A.

${ }^{l}$ now at University of Toronto, Dept of Physics, Toronto, Canada 
${ }^{m}$ current address Bergische Universität, Wuppertal, Germany

${ }^{n}$ and University of Mining and Metallurgy, Cracow, Poland

${ }^{o}$ now at University of California, San Diego, U.S.A.

${ }^{p}$ now at Physics Dept Southern Methodist University, Dallas, TX 75275, U.S.A.

${ }^{q}$ now at IPHE Université de Lausanne, CH-1015 Lausanne, Switzerland

${ }^{r}$ now at IEKP Universität Karlsruhe, Germany

${ }^{s}$ now at Universitaire Instelling Antwerpen, Physics Department, B-2610 Antwerpen, Belgium

${ }^{t}$ now at RWTH Aachen, Germany 


\section{Introduction}

Semileptonic B decays to orbitally-excited P-wave charm mesons $\left(\mathrm{D}^{* *}\right)$ are of interest for several reasons. Firstly, they constitute a significant fraction of B semileptonic decays, thereby accounting for some of the difference between the inclusive measurements and the sum of the exclusive B decay modes to $\mathrm{D}^{*} \ell \bar{\nu}$ and $\mathrm{D} \ell \bar{\nu}$ 3,1,2. They also contribute the major source of systematic error in the $\left|V_{c b}\right|$ measurement at LEP, as a background to the direct decay $\overline{\mathrm{B}}^{0} \rightarrow \mathrm{D}^{*+} \ell^{-} \bar{\nu}$. Finally, the measured decay properties can be compared with theoretical HQET predictions [4,1].

The $\mathrm{D}^{* *}$ mesons (sometimes denoted $\mathrm{D}_{J}$ ) are composed of a charm quark and a light quark in a state of orbital angular momentum $L=1$. In the infinite heavy-quark (charm) mass limit the $\mathrm{D}^{* *}$ system is equivalent to a fixed force center one-body problem. Hence the total (spin+orbital) angular momentum of the light quark degrees of freedom (labelled by $J_{q}=\frac{1}{2}$ or $\frac{3}{2}$ ) and the spin of the heavy quark are taken as separately conserved [1, 5]. The $J_{q}=\frac{3}{2}$ states combine with the heavy quark spin to form two states with $J^{P}=1^{+}\left(\mathrm{D}_{1}\right)$ and $J^{P}=2^{+}\left(\mathrm{D}_{2}^{*}\right)$. In the infinite charm mass limit, they can only undergo D-wave decay, and therefore have narrow widths [5]. For $J_{q}=\frac{1}{2}$ we have two states with $J^{P}=0^{+}$and $J^{P}=1^{+}$. The $J_{q}=\frac{1}{2}$ states can decay via $\mathrm{S}$-wave and are expected to be broad, but their experimental observation is still not established [3]. So in total, for P-wave mesons, four charged and four neutral $\mathrm{D}^{* *}$ states are predicted. Table 1 summarises the properties of the neutral states.

In this paper we present a new measurement of semileptonic B decays into the narrow neutral $\mathrm{D}_{1}^{0}$ and $\mathrm{D}_{2}^{* 0}$ states. We reconstruct events compatible with the decay chain

$$
\mathrm{b} \rightarrow \overline{\mathrm{B}} \rightarrow \mathrm{D}^{* * 0} \ell^{-} \bar{\nu} X, \mathrm{D}^{* * 0} \rightarrow \mathrm{D}^{*+} \pi^{-}, \mathrm{D}^{*+} \rightarrow \mathrm{D}^{0} \pi^{+}, \mathrm{D}^{0} \rightarrow(\mathrm{K} \pi \text { or } \mathrm{K} 3 \pi) .
$$

Here, and throughout this paper, $\overline{\mathrm{B}}$ refers to the $\overline{\mathrm{B}}^{0}$ and $\mathrm{B}^{-}$mesons, and charge conjugate modes and reactions are always implied. Also, $\ell$ refers to both electrons and muons, and the terms "kaon" and "pion" denote the charged particles.

These results update a previous OPAL analysis [7. Similar measurements have been performed also by ALEPH [2], DELPHI [8, CLEO [9] and ARGUS [10]. We note the complementarity between the LEP and $\Upsilon(4 \mathrm{~S})$ analyses, as in the latter, theoretical uncertainties in the B semileptonic decay form factors contribute significantly to the overall systematic error. This is less important at LEP, due to the high boost of the B hadron and its decay products.

\section{Detector and Monte Carlo Samples}

The OPAL detector is fully described elsewhere [11. A brief description of the main components relevant for this analysis follows. Tracking of charged particles is performed by a central detector, consisting of a silicon microvertex detector, a vertex chamber, a jet chamber and $z$-chambers ${ }^{1}$. The central detector is inside a solenoid, which provides a uniform axial magnetic field of $0.435 \mathrm{~T}$. The silicon microvertex detector consists of two layers of silicon strip detectors; for most of the data used in this paper, the inner layer covered a polar angle range of $|\cos \theta|<0.83$ and the outer layer covered $|\cos \theta|<0.77$, with an extended coverage for the data taken after 1996 . This detector provided only $\phi$-coordinate information for data taken in 1991-1992, and also $z$-coordinate information thereafter. The vertex chamber is a precision drift chamber which covers the range $|\cos \theta|<0.95$. The jet chamber is a large-volume drift chamber, $4.0 \mathrm{~m}$ long

\footnotetext{
${ }^{1} \mathrm{~A}$ right handed coordinate system is used, with positive $z$ along the $\mathrm{e}^{-}$beam direction and $x$ pointing towards the center of the LEP ring. The polar and azimuthal angles are denoted by $\theta$ and $\phi$, and the origin is taken to be the center of the detector.
} 
and $3.7 \mathrm{~m}$ in diameter, providing both tracking and ionisation energy loss $(\mathrm{d} E / \mathrm{d} x)$ information. The $z$-chambers measure the $z$-coordinate of tracks as they leave the jet chamber in the range $|\cos \theta|<0.72$. Immediately outside the tracking volume is the solenoid and a time-of-flight counter array followed by an electromagnetic shower presampler and a lead-glass electromagnetic calorimeter. The return yoke of the magnet lies outside the electromagnetic calorimeter and is instrumented with limited streamer chambers and thin gap chambers. It is used as a hadron calorimeter and assists in the reconstruction of muons. The outermost part of the detector is made up by layers of muon chambers.

A Monte Carlo simulation of hadronic $\mathrm{Z}$ decays of about five times the size of the recorded data sample for $\mathrm{b}$ flavoured events, and about the same size as the recorded data for the other flavours, is used in the analysis. In addition, signal Monte Carlo samples were generated with approximately one thousand times the expected number of signal events in the data. The simulated events were generated using JETSET 7.4 [12] with the b and c quark fragmentation modelled according to the parameterisation of Peterson et al. [13. A global fit to OPAL data has been performed to optimise the JETSET parameters [14. These events were processed using the full OPAL detector simulation [15] and analysed in the same manner as the data.

\section{Event Selection}

This analysis identifies decays compatible with the decay chain of Eq. 1 illustrated in Fig. 1] We attempt to identify the 5 relevant tracks in the case of the $\mathrm{D}^{0} \rightarrow \mathrm{K} \pi$ mode, or 7 tracks for the $\mathrm{D}^{0} \rightarrow \mathrm{K} 3 \pi$ mode, that match the correct event topology. To distinguish between the different charged pions involved, we denote as $\pi^{* *}$ the one coming from the $\mathrm{D}^{* * 0}$ decay. Similarly, $\pi_{\text {slow }}$ comes from the $\mathrm{D}^{*+}$ meson, its name arising from the small mass difference $\left(\Delta m^{*}\right)$ between the $\mathrm{D}^{*+}$ and the $\mathrm{D}^{0}$, leading to a very low pion momentum in the $\mathrm{D}^{*+}$ rest frame. Event selection criteria are applied to the data and Monte Carlo samples in five stages, namely hadronic Z preselection, reconstruction of $\mathrm{D}^{*+} \ell^{-}$candidates, identification of the best $\pi^{* *}$, selection of the best overall candidate, and final background suppression cuts specific to the $\mathrm{D}^{0} \rightarrow \mathrm{K} \pi$ and $\mathrm{D}^{0} \rightarrow \mathrm{K} 3 \pi$ modes.

\subsection{Hadronic Z Decay Preselection}

Hadronic Z decays collected with the OPAL detector at $\mathrm{e}^{+} \mathrm{e}^{-}$centre-of-mass energies near the Z resonance are selected using a standard OPAL hadronic event selection [16, with an additional requirement of at least 7 tracks per event. All the tracks must pass a set of standard quality cuts [17. The selection efficiency for hadronic $\mathrm{Z}$ decays is $(98.1 \pm 0.5) \%$ [17] with a background of $(0.11 \pm 0.03) \%$. Only data that were taken with the silicon microvertex detector in operation are used in this analysis. After this preselection, the resulting data sample collected in the years 1991-2000 consists of 3904417 events selected from a total integrated luminosity of $180.8 \mathrm{pb}^{-1}$ collected at the $\mathrm{Z}$ resonance. About $11 \%$ of the data used were recorded in the years 1996-2000.

\subsection{Reconstruction of $\mathrm{D}^{*+} \ell^{-}$Candidates}

Candidate events must have two or more jets, defined using a cone algorithm [18], with radius of 0.7 radians and $10 \mathrm{GeV}$ minimum energy. The primary vertex of the event is reconstructed using a beam spot constraint [19. We then identify muon or electron candidates with momentum greater than 3 or $2 \mathrm{GeV}$, respectively. The muon cut is more stringent to reduce the background 
from pions misidentified as muons. An artificial neural network is used to identify electrons [20, and photon conversions are rejected as described in [21]. Muons are selected as described in [17].

Next, we look for tracks consistent with the $\mathrm{D}^{*+}$ hypothesis and within the same jet as the lepton. Firstly, a kaon candidate with the same charge sign as the lepton, and momentum greater than $1.0 \mathrm{GeV}$, is required to have a $\mathrm{d} E / \mathrm{d} x$ probability greater than $1 \%$ for the kaon hypothesis. This requirement is increased to $10 \%$ if the $\mathrm{D}^{*+}$ energy is smaller than half the beam energy, where $\mathrm{K} / \pi$ separation is more powerful. Also, the measured $\mathrm{d} E / \mathrm{d} x$ of the kaon candidate is required to be smaller than the expected $\mathrm{d} E / \mathrm{d} x$ for the pion hypothesis.

A pion of opposite charge is then sought in order to form a $\mathrm{D}^{0} \rightarrow \mathrm{K} \pi$ candidate; for $\mathrm{D}^{0} \rightarrow \mathrm{K} 3 \pi$, two additional (pion) tracks of opposite charge are identified. The reconstructed $\mathrm{D}^{0}$ mass peak has a resolution of about $25 \mathrm{MeV}$; a loose cut of $1.79<M\left(\mathrm{D}^{0}\right)<1.94 \mathrm{GeV}$ is applied. A $\pi_{\text {slow }}$ track candidate of opposite charge to the kaon is combined with the $\mathrm{D}^{0}$ to form a $\mathrm{D}^{*+}$. The mass difference $\Delta m^{*} \equiv M\left(\mathrm{D}^{*+}\right)-M\left(\mathrm{D}^{0}\right)$ forms a sharp peak of about $1 \mathrm{MeV}$ resolution, and we require $140.5<\Delta m^{*}<149.5 \mathrm{MeV}$ at this stage. The energy of the $\mathrm{D}^{*+}$ is required to be greater than $15 \%$ of the beam energy. A $\mathrm{D}^{0}$ vertex is required to be successfully reconstructed using the 2 (or 4 ) candidate tracks. A kinematic fit performed on the $\mathrm{D}^{*+}$ tracks using the known $\mathrm{D}^{*+}$ and $\mathrm{D}^{0}$ masses as constraints is required to converge. We note that at this point, several different $\mathrm{D}^{*+} \ell^{-}$candidates per event can exist.

\subsection{Selection of Best Overall Candidate}

We now proceed to select the best $\pi^{* *}$ candidate for a given $\mathrm{D}^{*+} \ell^{-}$assignment, where $\pi^{* *}$ candidates are required at this stage to have an energy greater than $0.6 \mathrm{GeV}$ and the same charge as the lepton. A B vertex is constructed using the tracks of the $\pi^{* *}, \pi_{\text {slow }}$, lepton, and reconstructed $\mathrm{D}^{0}$. The primary vertex is then recomputed, excluding all of the above 5 (or 7 ) tracks. The new $\chi^{2}$ of the primary vertex is obtained, and for each $\mathrm{D}^{*+} \ell^{-}$combination the $\pi^{* *}$ candidate that gives the smallest combined $\chi^{2}$ of the primary and $\mathrm{B}$ vertices is chosen. The $\mathrm{B}$ and $\mathrm{D}$ decay lengths are recomputed with the new vertex positions. In order to select only well reconstructed B vertices, we require an estimated uncertainty of less than $1 \mathrm{~mm}$ on the B decay length.

Having selected the best $\pi^{* *}$ candidate for the given $\mathrm{D}^{*+} \ell^{-}$assignment, we reconstruct the mass difference $\Delta m^{* *} \equiv M\left(\mathrm{D}^{* * 0}\right)-M\left(\mathrm{D}^{*+}\right)$, and restrict it to the range $0.14-1.10 \mathrm{GeV}$. We then select for further analysis only one $\mathrm{D}^{*+} \ell^{-}$candidate per event, namely the one with highest $\mathrm{D}^{*+}$ kinematic fit probability. This cut greatly reduces the combinatorial background arising from fake combinations when forming the $\mathrm{D}^{*+} \ell^{-}$.

\subsection{Final Cuts}

The main backgrounds at this point arise from fragmentation, where a pion from the $\mathrm{b}$ quark fragmentation fakes a $\pi^{* *}$ from a $\mathrm{D}^{* * 0}$, and combinatorial backgrounds; we include in the fragmentation category pions from $\mathrm{B}^{* *} \rightarrow \mathrm{B} \pi X$. The cuts for reducing these backgrounds differ somewhat for the two $\mathrm{D}^{0}$ decay modes, the $\mathrm{D}^{0} \rightarrow \mathrm{K} 3 \pi$ mode having larger backgrounds, and correspondingly stronger cuts.

To reduce the fragmentation background, we make the following requirements:

- The $\pi^{* *}$ energy must be greater than $1 \mathrm{GeV}$. 
- The output of a neural network applied to the $\pi^{* *}$ track is required to be greater than 0.6 for the $\mathrm{D}^{0} \rightarrow \mathrm{K} 3 \pi$ mode, and greater than 0.3 for the $\mathrm{D}^{0} \rightarrow \mathrm{K} \pi$ mode. This neural network uses momentum, transverse momentum to the jet, and impact parameter significance with respect to the primary vertex; it is used to distinguish between tracks from the primary vertex and genuine $\mathrm{B}$ decay tracks 22 .

- The $\mathrm{B}$ decay length significance $l_{\mathrm{B}} / \sigma_{l_{\mathrm{B}}}$ is required to be greater than 1.5 , where $l_{\mathrm{B}}$ is the signed $\mathrm{B}$ decay length and $\sigma_{l_{\mathrm{B}}}$ its error. It is signed positive if the $\mathrm{B}$ vertex is displaced from the primary vertex in the same direction as the jet momentum, and negative otherwise.

- Similarly, $l_{\mathrm{B}-\mathrm{D}} / \sigma_{l_{\mathrm{B}-\mathrm{D}}}>-2.0$ for the signed decay length significance between the $\mathrm{B}$ and $\mathrm{D}$ vertices. It is signed positive if the $\mathrm{D}$ vertex is displaced from the $\mathrm{B}$ vertex in the same direction as the jet momentum, and negative otherwise.

The combinatorial background is suppressed using the following cuts:

- $143<\Delta m^{*}<148 \mathrm{MeV}$ for $\mathrm{D}^{0} \rightarrow \mathrm{K} 3 \pi, 142<\Delta m^{*}<149 \mathrm{MeV}$ for $\mathrm{D}^{0} \rightarrow \mathrm{K} \pi$.

- $1.815<M\left(\mathrm{D}^{0}\right)<1.915 \mathrm{GeV}$ for $\mathrm{D}^{0} \rightarrow \mathrm{K} 3 \pi$.

- $\cos \theta^{*}>-0.9$, where $\theta^{*}$ is the angle between the kaon and the $\mathrm{D}^{0}$ boost direction, calculated in the $\mathrm{D}^{0}$ rest frame. This uses the fact that the $\mathrm{D}^{0}$ is a pseudoscalar, whereas the background, particularly that resulting from particle misidentification, tends to peak at large negative values of $\cos \theta^{*}$.

- For the $\mathrm{D}^{0} \rightarrow \mathrm{K} 3 \pi$ mode, the product of the neural network applied to the 4 tracks from the $\mathrm{D}^{0}$ decay, is required to be greater than 0.2 .

Fake leptons and non- $\overline{\mathrm{B}}$ semileptonic decays appear at a smaller rate than the previous backgrounds, but their invariant mass distributions can peak in the expected signal region. The non- $\overline{\mathrm{B}}$ semileptonic background arises primarily from charm, tau and b-baryon decays, and pion misidentification. Therefore we impose the following additional cuts:

- The $\mathrm{D}^{*+} \ell^{-}$invariant mass is restricted to the range $2.8-5.0 \mathrm{GeV}$.

- The lepton momentum transverse to the jet: $\mathrm{p}_{\mathrm{T}, \ell}>0.9 \mathrm{GeV}$ for $\mathrm{D}^{0} \rightarrow \mathrm{K} 3 \pi$, and $>0.6 \mathrm{GeV}$ for $\mathrm{D}^{0} \rightarrow \mathrm{K} \pi$. This cut is effective in reducing misidentified lepton backgrounds and does not reduce the signal significance.

These selection criteria were designed to maximise the efficiency $\times$ purity using only quantities well modelled by the Monte Carlo simulation. As an example, Fig. 2 presents the data versus Monte Carlo distributions after all cuts for four of the most relevant quantities used in the analysis. Since the final analysis consists of searches for peaks in the distribution of $\Delta m^{* *}$, the cuts were also tuned so that the simulated background $\Delta m^{* *}$ distribution shows no peak in the signal region, $0.35<\Delta m^{* *}<0.55 \mathrm{GeV}$.

\section{Backgrounds and Wrong Sign Sample}

Applying the previous selection to our combined $\mathrm{D}^{0} \rightarrow(\mathrm{K} \pi$ or $\mathrm{K} 3 \pi)$ Monte Carlo samples, the major background process is $\overline{\mathrm{B}}^{0} \rightarrow \mathrm{D}^{*+} \ell^{-} \bar{\nu}$, where the $\mathrm{D}^{*+}$ is combined with an additional pion of the right sign. This comprises $65 \%$ of the total background. The decays $\overline{\mathrm{B}} \rightarrow \mathrm{D}^{* * 0} \ell^{-} \bar{\nu} X$ and $\overline{\mathrm{B}} \rightarrow \mathrm{D}^{* *+} \ell^{-} \bar{\nu} X$, where some of the tracks are incorrectly matched, amount to $16 \%$ (using the branching fractions measured in this paper). These three distinct physical processes have in common that the $\pi^{* *}$ track is incorrectly identified, and therefore they constitute the major part 
of the above mentioned fragmentation background. The kinematics of this dominant background should be equivalent to the one obtained by requiring a $\pi^{* *}$ of the opposite charge from that expected for $\mathrm{D}^{* * 0}$ decay. The Monte Carlo $\Delta m^{* *}$ distributions for background right sign and wrong sign $\pi^{* *}$ are shown in Fig. 3a,b. They are fitted to a functional form $x^{\gamma} e^{-\beta x}$ where $x \equiv \Delta m^{* *}-m_{\pi}$; the fitted values of the $\gamma$ and $\beta$ parameters for the two samples are consistent. Fig. 3r shows the OPAL data wrong sign $\Delta m^{* *}$ distribution; the fitted $\gamma$ and $\beta$ parameters agree with those obtained in Monte Carlo.

Fake leptons, $\mathrm{B}_{\mathrm{s}}$, and non- $\overline{\mathrm{B}}$ semileptonic decays constitute less severe backgrounds amounting to $3 \%, 5 \%$ and $11 \%$ of the total background, respectively. We find the contribution from $\mathrm{Z}$ decays into charm and lighter quarks to be negligible.

\section{$5 \quad$ Signal Fitting Procedure}

An unbinned maximum likelihood fit is performed simultaneously on the $\Delta m^{* *}$ right sign and wrong sign distributions. For the right sign fit, the function is given by the sum of two BreitWigner distributions, each convolved with a Gaussian resolution function, plus the background function $x^{\gamma} e^{-\beta x}$ described previously; for the wrong sign fit, only the background function is used. There are six fitted parameters: the number of $D_{1}^{0}$ and $D_{2}^{* 0}$, the normalisation of the right sign and wrong sign backgrounds, and the background shape parameters $\gamma$ and $\beta$. Thus, the background shape and normalisation are obtained directly from data. We fix the narrow states masses and widths to the world averages [3], shown in Table 1] The detector resolution on the reconstructed $\Delta m^{* *}$ is fixed at $\sigma=8 \mathrm{MeV}$, as obtained from Monte Carlo.

To check for biases and to assess the systematic uncertainties, the fitting procedure was tested by comparing the results of five simulated experiments, each one with the same statistics as the data (so-called "ensemble tests"). The pull distributions for the fitted number of $\mathrm{D}_{1}^{0}, \mathrm{D}_{2}^{* 0}$, and background events were found to be consistent with zero mean and unit variance for both the $\mathrm{D}^{0}$ decay modes taken separately as well as when fitting to the two modes combined. The uncertainty is taken to be the error on the mean of the pull distributions. We note that the two narrow peaks are resolvable and we are able to correctly fit the number of $\mathrm{D}_{1}^{0}$ and $\mathrm{D}_{2}^{* 0}$ signal events in the Monte Carlo simulations for various input signal branching ratios.

\section{Results}

The final $\Delta m^{* *}$ distributions for the $\mathrm{D}^{0} \rightarrow \mathrm{K} \pi$ and $\mathrm{D}^{0} \rightarrow \mathrm{K} 3 \pi$ modes are shown in Fig. 4. The numerical results of the fit are also shown, including the $1 \sigma$ uncertainties on the fitted parameters. A signal for the expected narrow $\mathrm{D}_{1}^{0}$ peak is present in each mode separately, with a weaker significance for the $\mathrm{D}^{0} \rightarrow \mathrm{K} \pi$ mode (8.0 45.0 fitted $\mathrm{D}_{1}^{0}$ events) than for $\mathrm{D}^{0} \rightarrow \mathrm{K} 3 \pi$ (21.4 \pm 7.2 fitted $\mathrm{D}_{1}^{0}$ events). No evidence of a $\mathrm{D}_{2}^{* 0}$ signal is present.

The efficiencies estimated for the $\mathrm{D}_{1}^{0}$ state using dedicated signal simulations are found to be $7.7 \pm 0.4 \%$ and $2.3 \pm 0.1 \%$ for the $\mathrm{D}^{0} \rightarrow \mathrm{K} \pi$ and $\mathrm{D}^{0} \rightarrow \mathrm{K} 3 \pi$ modes, respectively, where the errors are statistical only. For the $\mathrm{D}_{2}^{* 0}$ state these are $9.2 \pm 0.8 \%$ and $2.1 \pm 0.2 \%$. The product branching ratio can then be obtained as:

$$
\begin{gathered}
\mathrm{BR}(\mathrm{b} \rightarrow \overline{\mathrm{B}}) \times \mathrm{BR}\left(\overline{\mathrm{B}} \rightarrow \mathrm{D}^{* * 0} \ell^{-} \bar{\nu} X\right) \times \mathrm{BR}\left(\mathrm{D}^{* * 0} \rightarrow \mathrm{D}^{*+} \pi^{-}\right)= \\
\frac{N_{\mathrm{Fit}}\left(\mathrm{D}^{* * 0}\right) / \varepsilon_{\mathrm{D}^{* * 0}}}{\left(N_{\mathrm{Z}} / \varepsilon_{\mathrm{Z}}\right) \times R_{\mathrm{b}} \times 2 \times 2 \times \mathrm{BR}\left(\mathrm{D}^{*+} \rightarrow \mathrm{D}^{0} \pi^{+}\right) \times \mathrm{BR}\left(\mathrm{D}^{0} \rightarrow \mathrm{K} \pi, \mathrm{K} 3 \pi\right)}
\end{gathered}
$$


The two factors of 2 arise from the two b hadrons in the event, and the two flavors of tagged lepton. The fraction, $R_{\mathrm{b}}$, of $\mathrm{Z} \rightarrow \mathrm{b} \overline{\mathrm{b}}$ events in $\mathrm{Z}$ hadronic decays, and the branching ratios for $\mathrm{D}^{*+} \rightarrow \mathrm{D}^{0} \pi^{+}$and $\mathrm{D}^{0} \rightarrow \mathrm{K} \pi$ are taken from [3]; $N_{\mathrm{Z}}=3904417$ and $\varepsilon_{\mathrm{Z}}=(98.1 \pm 0.5) \%$, as mentioned in section 3.1 .

For decays into the $\mathrm{D}_{1}^{0}$ state, we obtain for the $\mathrm{D}^{0} \rightarrow \mathrm{K} \pi$ mode:

$$
\mathrm{BR}(\mathrm{b} \rightarrow \overline{\mathrm{B}}) \times \mathrm{BR}\left(\overline{\mathrm{B}} \rightarrow \mathrm{D}_{1}^{0} \ell^{-} \bar{\nu} X\right) \times \mathrm{BR}\left(\mathrm{D}_{1}^{0} \rightarrow \mathrm{D}^{*+} \pi^{-}\right)=(1.17 \pm 0.73 \pm 0.27) \times 10^{-3}
$$

and for $\mathrm{D}^{0} \rightarrow \mathrm{K} 3 \pi$ :

$$
\mathrm{BR}(\mathrm{b} \rightarrow \overline{\mathrm{B}}) \times \mathrm{BR}\left(\overline{\mathrm{B}} \rightarrow \mathrm{D}_{1}^{0} \ell^{-} \bar{\nu} X\right) \times \mathrm{BR}\left(\mathrm{D}_{1}^{0} \rightarrow \mathrm{D}^{*+} \pi^{-}\right)=(5.30 \pm 1.79 \pm 0.95) \times 10^{-3}
$$

where the first error is statistical and the second systematic. The evaluation of the systematic uncertainty is discussed in section [7. The two product branching ratios measured for the $\mathrm{D}^{0} \rightarrow \mathrm{K} \pi$ and $\mathrm{D}^{0} \rightarrow \mathrm{K} 3 \pi$ decay modes agree at the level of $1.9 \sigma$.

The two modes were combined by merging the two data samples and performing a single fit, which has the advantage of reducing the systematic error on the background. The resulting distribution is shown in Fig [5. The combined efficiency, multiplied by the respective $\mathrm{D}^{0}$ decay branching ratios, is $0.47 \%(7.7 \% \times 3.80 \%+2.3 \% \times 7.46 \%)$. There are $28.7 \pm 8.6$ events in the $\mathrm{D}_{1}^{0}$ peak, from which the product branching ratio follows:

$$
\mathrm{BR}(\mathrm{b} \rightarrow \overline{\mathrm{B}}) \times \mathrm{BR}\left(\overline{\mathrm{B}} \rightarrow \mathrm{D}_{1}^{0} \ell^{-} \bar{\nu} X\right) \times \mathrm{BR}\left(\mathrm{D}_{1}^{0} \rightarrow \mathrm{D}^{*+} \pi^{-}\right)=(2.64 \pm 0.79 \pm 0.39) \times 10^{-3} .
$$

The combined-sample fit yields $3.1 \pm 6.9$ events for the $\mathrm{D}_{2}^{* 0}$. The measured branching ratio is then consistent with zero:

$$
\mathrm{BR}(\mathrm{b} \rightarrow \overline{\mathrm{B}}) \times \mathrm{BR}\left(\overline{\mathrm{B}} \rightarrow \mathrm{D}_{2}^{* 0} \ell^{-} \bar{\nu} X\right) \times \mathrm{BR}\left(\mathrm{D}_{2}^{* 0} \rightarrow \mathrm{D}^{*+} \pi^{-}\right)=(0.26 \pm 0.59 \pm 0.35) \times 10^{-3} .
$$

Using the method of Feldman and Cousins [23, 3], this can be converted into a 95\% confidence level upper limit:

$$
\mathrm{BR}(\mathrm{b} \rightarrow \overline{\mathrm{B}}) \times \mathrm{BR}\left(\overline{\mathrm{B}} \rightarrow \mathrm{D}_{2}^{* 0} \ell^{-} \bar{\nu} X\right) \times \mathrm{BR}\left(\mathrm{D}_{2}^{* 0} \rightarrow \mathrm{D}^{*+} \pi^{-}\right)<1.39 \times 10^{-3} .
$$

These results were checked in several ways. Firstly, if instead of fixing the $\mathrm{D}_{1}^{0}$ mass we include it as an extra parameter to be fitted we obtain:

$$
\mathrm{BR}(\mathrm{b} \rightarrow \overline{\mathrm{B}}) \times \mathrm{BR}\left(\overline{\mathrm{B}} \rightarrow \mathrm{D}_{1}^{0} \ell^{-} \bar{\nu} X\right) \times \mathrm{BR}\left(\mathrm{D}_{1}^{0} \rightarrow \mathrm{D}^{*+} \pi^{-}\right)=(2.66 \pm 0.80 \pm 0.39) \times 10^{-3},
$$

in agreement with the previous result of $(2.64 \pm 0.79 \pm 0.39) \times 10^{-3}$. The fitted $\mathrm{D}_{1}^{0}$ mass is $(2418.8 \pm$ 5.0) $\mathrm{MeV}$, consistent with the world average of $(2422.2 \pm 1.9) \mathrm{MeV}$ [3. The cuts described in section 3.4 were varied by significant amounts (10\%-100\%), and the resulting variations in the measured branching ratios were found to be compatible with the expected statistical variations. To check the procedure for combining the results of the two $\mathrm{D}^{0}$ decay modes, we calculated a simple weighted average of the two results. This average is consistent with the combined result.

We also considered the angular decay distribution of the narrow states. In the heavy-quark limit, the pure D-wave decay of the $J^{P}=2^{+} \mathrm{D}_{2}^{* 0}$ state should be distributed according to $\frac{3}{4} \sin ^{2} \alpha$, whereas for the $J^{P}=1^{+} \mathrm{D}_{1}^{0}$ we expect $\frac{1}{4}\left(1+3 \cos ^{2} \alpha\right)[\underline{5}$. This has been observed experimentally by CLEO 24]. Here $\alpha$ is the angle between the $\pi^{* *}$ and the $\pi_{\text {slow }}$ from the $\mathrm{D}^{*+}$ decay, evaluated in the rest frame of the $\mathrm{D}^{*+}$. For $\mathrm{D}_{1}^{0}$ events we expect more events at larger values of $|\cos \alpha|$, and this is evident in Fig. 6a which shows this angle for data and Monte Carlo in the signal mass region $0.35<\Delta m^{* *}<0.55 \mathrm{GeV}$. The shape of the data distribution 
is observed to agree with that expected from simulated events, where the signal rate is fixed to the one measured here. Fig. 6b shows that background events, selected from right sign data in the sidebands $0.14<\Delta m^{* *}<0.3$ or $0.6<\Delta m^{* *}<1.1 \mathrm{GeV}$, exhibit a flat $\cos \alpha$ distribution. An enhancement of the $D_{1}^{0}$ resonance peak is expected for higher values of $|\cos \alpha|$, and this is confirmed in Fig. 6. as the $\Delta m^{* *} \mathrm{D}_{1}^{0}$ peak is enhanced with a selection $|\cos \alpha|>0.5$. Note how the overall level of the background drops relative to Fig. 5 . Conversely, for $|\cos \alpha|<0.5$ (Fig. 6 $\mathrm{d}$ ), the $\mathrm{D}_{1}^{0}$ peak greatly diminishes in significance.

For a $\mathrm{D}_{2}^{* 0}$ enhancement selection, namely $|\cos \alpha|<0.2$, we find no distinct sharp peak at the expected $\mathrm{D}_{2}^{* 0}$ position. This provides additional evidence for the presence of the $\mathrm{D}_{1}^{0}$ state and absence of the $\mathrm{D}_{2}^{* 0}$.

\section{Systematic Uncertainties}

The systematic uncertainties in the product branching ratios are shown in Table 2. The dominant contributions arise from uncertainties in the background function and the $\mathrm{D}_{1}^{0}$ and $\mathrm{D}_{2}^{* 0}$ fit parameters. The components of systematic uncertainty shown in the table are:

- Background function: The dominant uncertainty on the background arises from the possibility that some background has a peak in the signal region, therefore biasing the fitted number of $\mathrm{D}_{1}^{0}$ and $\mathrm{D}_{2}^{* 0}$ signal events. This uncertainty is estimated from the average number of (expected-fitted) signal events, as obtained in the Monte Carlo ensemble tests described in Section [5. There is also a much smaller background uncertainty on the overall background shape. This component of the uncertainty is estimated by comparing the $\gamma, \beta$ parameters obtained from Monte Carlo with those obtained from the wrong sign $\pi^{* *}$ data sample.

- $\mathrm{D}^{* * 0}$ fit parameters: We vary the $\mathrm{D}_{1}^{0}$ and $\mathrm{D}_{2}^{* 0}$ masses and widths within the current uncertainties [3], and refit the $\Delta m^{* *}$ distribution. We also vary the Gaussian resolution by $\pm 1 \mathrm{MeV}$ and redo the fit. The resulting variations are added in quadrature.

All the remaining sources of systematic uncertainty affect only the signal efficiency, as the background shape and normalisation are obtained directly from the data.

- The limited Monte Carlo statistics in the signal samples give rise to a systematic uncertainty on the estimation of efficiencies.

- The lepton identification efficiency has an uncertainty of $3 \%$ for muons and $4 \%$ for electrons [25.

- The mean and sigma of the normalised Monte Carlo $\mathrm{d} E / \mathrm{d} x$ distributions were varied by $\pm 10 \%[26$.

- Tracking resolution: The systematic uncertainty was assessed in Monte Carlo by applying a global 10\% degradation to the resolution of all measured track parameters.

- The lifetime of the $\bar{B}$ mesons was varied within their measured uncertainty 3 .

- Theoretical uncertainty in the $\overline{\mathrm{B}} \rightarrow \mathrm{D}^{* * 0} \ell^{-} \bar{\nu} X$ form factors: Different theoretical models predict different form factors for the $\mathrm{B}$ semileptonic decay, and therefore different $\pi^{* *}$ 
energy spectra. This source of systematic uncertainty is dominant at the $\Upsilon(4 \mathrm{~S})$ experiments 9]. Due to the high boost provided in $\mathrm{Z} \rightarrow \mathrm{b} \overline{\mathrm{b}}$ decays, this uncertainty is expected to be significantly smaller in the analyses at LEP. This was confirmed by reweighting our JETSET [12] Monte Carlo samples to the forms factors described in [27]. The range of parameters describing the form factor calculation was varied within the values constrained as in [28], and also the values needed to approximately reproduce the form factors predicted by 29. The maximum variations in the signal efficiency were taken.

- B fragmentation: The Peterson fragmentation model [13] is used in JETSET [12] to model the momentum distribution of the B hadrons. Our Monte Carlo samples were reweighted event by event to reproduce the experimental uncertainty on the mean energy of the $\mathrm{B}$ hadrons. The Peterson parameter $\varepsilon_{\mathrm{b}}$ was varied within the range obtained in [30. The variations are in agreement with those obtained using two other fragmentation models [31, 32,33, again with parameter ranges as determined in [30.

- The relevant branching ratios and $R_{\mathrm{b}}$ were varied within the published uncertainties [3].

- The hadronic Z preselection efficiency was varied by its uncertainty of $\pm 0.5 \%$ [17.

\section{Conclusions}

We have analysed semileptonic $\mathrm{B}$ decays into the narrow $\mathrm{D}_{1}^{0}$ and $\mathrm{D}_{2}^{* 0}$ states in events compatible with the decay chain $\mathrm{b} \rightarrow \overline{\mathrm{B}} \rightarrow \mathrm{D}^{* * 0} \ell^{-} \bar{\nu} X, \mathrm{D}^{* * 0} \rightarrow \mathrm{D}^{*+} \pi^{-}, \mathrm{D}^{*+} \rightarrow \mathrm{D}^{0} \pi^{+}, \mathrm{D}^{0} \rightarrow(\mathrm{K} \pi$ or K $3 \pi)$. The product branching ratio for decays into the $\mathrm{D}_{1}^{0}$ state is measured to be:

$$
\mathrm{BR}(\mathrm{b} \rightarrow \overline{\mathrm{B}}) \times \mathrm{BR}\left(\overline{\mathrm{B}} \rightarrow \mathrm{D}_{1}^{0} \ell^{-} \bar{\nu} X\right) \times \mathrm{BR}\left(\mathrm{D}_{1}^{0} \rightarrow \mathrm{D}^{*+} \pi^{-}\right)=(2.64 \pm 0.79 \pm 0.39) \times 10^{-3},
$$

where the first error is statistical and the second systematic. We find no evidence for decays into the $J^{P}=2^{+}$state, and set a limit on the product branching ratio:

$$
\mathrm{BR}(\mathrm{b} \rightarrow \overline{\mathrm{B}}) \times \mathrm{BR}\left(\overline{\mathrm{B}} \rightarrow \mathrm{D}_{2}^{* 0} \ell^{-} \bar{\nu} X\right) \times \mathrm{BR}\left(\mathrm{D}_{2}^{* 0} \rightarrow \mathrm{D}^{*+} \pi^{-}\right)<1.4 \times 10^{-3},
$$

at the $95 \%$ confidence level. These results update a previous OPAL analysis [7, and agree with similar measurements performed at LEP [2,8, CLEO 9], and ARGUS [10].

\section{Acknowledgements}

We particularly wish to thank the SL Division for the efficient operation of the LEP accelerator at all energies and for their close cooperation with our experimental group. In addition to the support staff at our own institutions we are pleased to acknowledge

Support from the scholarship PRAXIS/BD/11460/97, Portugal, Department of Energy, USA, National Science Foundation, USA, Particle Physics and Astronomy Research Council, UK, Natural Sciences and Engineering Research Council, Canada, Israel Science Foundation, administered by the Israel Academy of Science and Humanities, Benoziyo Center for High Energy Physics,

Japanese Ministry of Education, Culture, Sports, Science and Technology (MEXT) and a grant under the MEXT International Science Research Program, 
Japanese Society for the Promotion of Science (JSPS),

German Israeli Bi-national Science Foundation (GIF),

Bundesministerium für Bildung und Forschung, Germany,

National Research Council of Canada,

Hungarian Foundation for Scientific Research, OTKA T-029328, and T-038240,

The NWO/NATO Fund for Scientific Reasearch, the Netherlands.

\section{References}

[1] J. Richman and P. Burchat, Rev. Mod. Phys. 67, 893 (1995).

[2] ALEPH Collaboration, D. Buskulic et al., Z. Phys. C73 (1997) 601.

[3] The Review of Particle Physics, K. Hagiwara et al., Phys. Rev. D66 (2002).

[4] M. Neubert, Phys. Reports 245, 259 (1994).

[5] J. Rosner, Comm. Nucl. Part. Phys. 62 (1986) 109.

[6] S. Godfrey and R. Kokoski, Phys. Rev. D43 (1991) 1679.

[7] OPAL Collaboration, R. Akers et al., Z. Phys. C67 (1995) 57.

[8] DELPHI Collaboration, P. Abreu et al., Phys. Lett. B457 (2000) 407.

[9] CLEO Collaboration, A. Anastassov et al., Phys. Rev. Lett.80 (1998) 4127.

[10] ARGUS Collaboration, H. Albrecht et al., Z. Phys. C57 (1993) 533.

[11] OPAL Collaboration, K. Ahmet et al., Nucl. Instr. Meth. A305 (1991) 275;

P.P. Allport et al., Nucl. Instr. Meth. A324 (1993) 34;

P.P. Allport et al., Nucl. Instr. Meth. A346 (1994) 476;

O. Biebel et al., Nucl. Instr. Meth. A323 (1992) 169;

M. Hauschild et al., Nucl. Instr. Meth. A314 (1992) 74;

S. Anderson et al., Nucl. Instr. Meth. A403 (1998) 326.

[12] T. Sjöstrand, Comp. Phys. Comm. 82 (1994) 74.

[13] C. Peterson, D. Schlatter, I. Schmitt and P. Zerwas, Phys. Rev. D27 (1983) 105.

[14] OPAL Collaboration, G. Alexander et al., Z. Phys. C69 (1996) 543.

[15] J. Allison et al., Nucl. Instr. Meth. A305 (1992) 47.

[16] OPAL Collaboration, G. Alexander et al., Z. Phys. C52 (1991) 175.

[17] OPAL Collaboration, R. Akers et al., Z. Phys. C65 (1995) 17.

[18] OPAL Collaboration, R. Akers et al., Z. Phys. C 63 (1994) 197.

[19] OPAL Collaboration, K. Ackerstaff et al., Z. Phys. C 74 (1997) 1.

[20] OPAL Collaboration, G. Alexander et al., Z. Phys. C 70 (1996) 357.

[21] OPAL Collaboration, P. D. Acton et al., Z. Phys. C 58 (1993) 523. 
[22] OPAL Collaboration, G. Abbiendi et al., Eur. Phys. J. C23 (2002) 437.

[23] G. Feldman and R. Cousins, Phys. Rev. D57 (1998) 3873.

[24] CLEO Collaboration, P. Avery et al., Phys. Lett. B331 (1994) 236.

[25] OPAL Collaboration, G. Abbiendi et al., Eur. Phys. J. C8 (1999) 217.

[26] OPAL Collaboration, G. Abbiendi et al., Eur. Phys. J. C21 (2001) 23.

[27] A. Leibovich, Z. Ligeti, I. Stewart and M. Wise, Phys. Rev. D57 (1998) 308.

[28] OPAL Collaboration, G. Abbiendi et al., Phys. Lett. B482 (2000) 15.

[29] D. Scora and N. Isgur, Phys. Rev. D52 (1995) 2783.

[30] OPAL Collaboration, G. Abbiendi et al., CERN-EP/2002-051, submitted to Eur. Phys. J. C.

[31] V.G. Kartvelishvili, A.K. Likhoded, V.A. Petrov, Phys. Lett. B78 (1978) 615.

[32] P.D.B. Collins and T.P. Spiller, J. Phys. G11 (1985) 1289.

[33] M.G. Bowler, Z. Phys. C11 (1981) 169. 


\begin{tabular}{|llllcl|}
\hline & $J^{P}$ & $J_{q}$ & $\begin{array}{l}\text { Mass } \\
(\mathrm{MeV})\end{array}$ & $\begin{array}{l}\text { Width } \\
(\mathrm{MeV})\end{array}$ & Decay Modes \\
\hline $\mathrm{D}_{1}^{\prime 0}$ & $1^{+}$ & $1 / 2$ & $\sim 2470$ & $\gtrsim 250$ & $\mathrm{D}^{*} \pi$ \\
$\mathrm{D}_{0}^{* 0}$ & $0^{+}$ & $1 / 2$ & $\sim 2400$ & $\gtrsim 170$ & $\mathrm{D} \pi$ \\
$\mathrm{D}_{1}^{0}(2420)$ & $1^{+}$ & $3 / 2$ & $2422.2 \pm 1.8$ & $18.9_{-3.5}^{+4.6}$ & $\mathrm{D}^{*} \pi$ \\
$\mathrm{D}_{2}^{* 0}(2460)$ & $2^{+}$ & $3 / 2$ & $2458.9 \pm 2.0$ & $23 \pm 5$ & $\mathrm{D} \pi, \mathrm{D}^{*} \pi$ \\
\hline
\end{tabular}

Table 1: Properties of neutral orbitally-excited charm mesons. The quantum number $J_{q}$ is the total angular momentum of the light constituents of the meson. Masses and widths of the narrow $J_{q}=3 / 2$ states are experimentally determined [3]. For the light $J_{q}=1 / 2$ states, we quote theoretical estimates of the masses and widths [6].

\begin{tabular}{|c|c|c|c|}
\hline \multirow[b]{2}{*}{ Source } & \multicolumn{3}{|c|}{ Systematic Uncertainty $\left(\times 10^{-3}\right)$} \\
\hline & $K \pi$ mode & $K 3 \pi$ mode & Combined \\
\hline Background function & \pm 0.23 & \pm 0.54 & \pm 0.17 \\
\hline $\mathrm{D}_{1}, \mathrm{D}_{2}$ fit parameters & $\begin{array}{l}+0.09 \\
+0.06\end{array}$ & $\begin{array}{l}+0.54 \\
+0.42\end{array}$ & ${ }_{-0.17}^{+0.22}$ \\
\hline Signal simulation statistics & $\begin{array}{l}-0.00 \\
\pm 0.06\end{array}$ & $\begin{array}{l}-0.42 \\
\pm 0.20\end{array}$ & \pm 0.12 \\
\hline Lepton ID & \pm 0.04 & \pm 0.18 & \pm 0.09 \\
\hline $\mathrm{d} E / \mathrm{d} x$ & \pm 0.05 & \pm 0.26 & \pm 0.11 \\
\hline Tracking resolution & \pm 0.04 & \pm 0.27 & \pm 0.10 \\
\hline B lifetime & \pm 0.01 & \pm 0.05 & \pm 0.03 \\
\hline Theoretical uncertainty & \pm 0.03 & \pm 0.16 & \pm 0.08 \\
\hline B fragmentation & \pm 0.04 & \pm 0.26 & \pm 0.10 \\
\hline$R_{\mathrm{b}}$ & \pm 0.004 & \pm 0.02 & \pm 0.009 \\
\hline $\mathrm{BR}\left(\mathrm{D}^{*+} \rightarrow \mathrm{D}^{0} \pi\right)$ & \pm 0.008 & \pm 0.04 & \pm 0.02 \\
\hline $\mathrm{BR}\left(\mathrm{D}^{0} \rightarrow \mathrm{K} \pi\right)$ & \pm 0.03 & & \pm 0.02 \\
\hline $\mathrm{BR}\left(\mathrm{D}^{0} \rightarrow \mathrm{K} 3 \pi\right)$ & & \pm 0.22 & \pm 0.13 \\
\hline Hadronic $\mathrm{Z}$ preselection & \pm 0.006 & \pm 0.03 & \pm 0.01 \\
\hline Total & $\begin{array}{l}+0.27 \\
-0.26\end{array}$ & $\begin{array}{l}+0.98 \\
-0.92\end{array}$ & $\begin{array}{l}+0.40 \\
-0.38\end{array}$ \\
\hline
\end{tabular}

Table 2: Systematic uncertainties on the product branching ratios. The combined column is for the combination resulting from merging the two data samples and performing a single fit. 


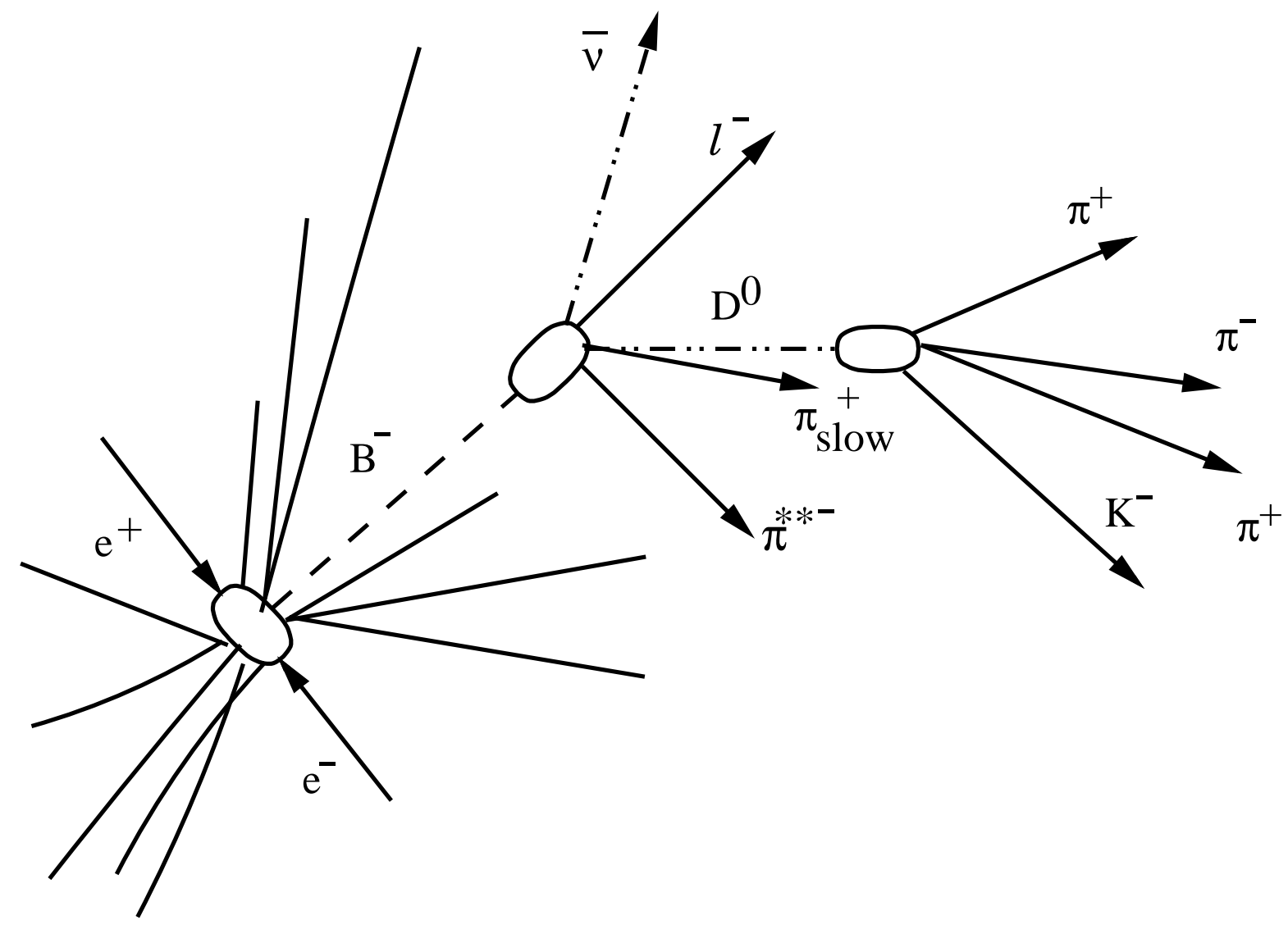

Figure 1: Event topology for a semileptonic $\mathrm{B}$ decay into a $\mathrm{D}^{* * 0} \ell^{-} \bar{\nu}, \mathrm{D}^{* * 0} \rightarrow \mathrm{D}^{*+} \pi^{-}$, $\mathrm{D}^{*+} \rightarrow \mathrm{D}^{0} \pi^{+}, \mathrm{D}^{0} \rightarrow \mathrm{K} 3 \pi$, with the 3 reconstructed vertices shown. 

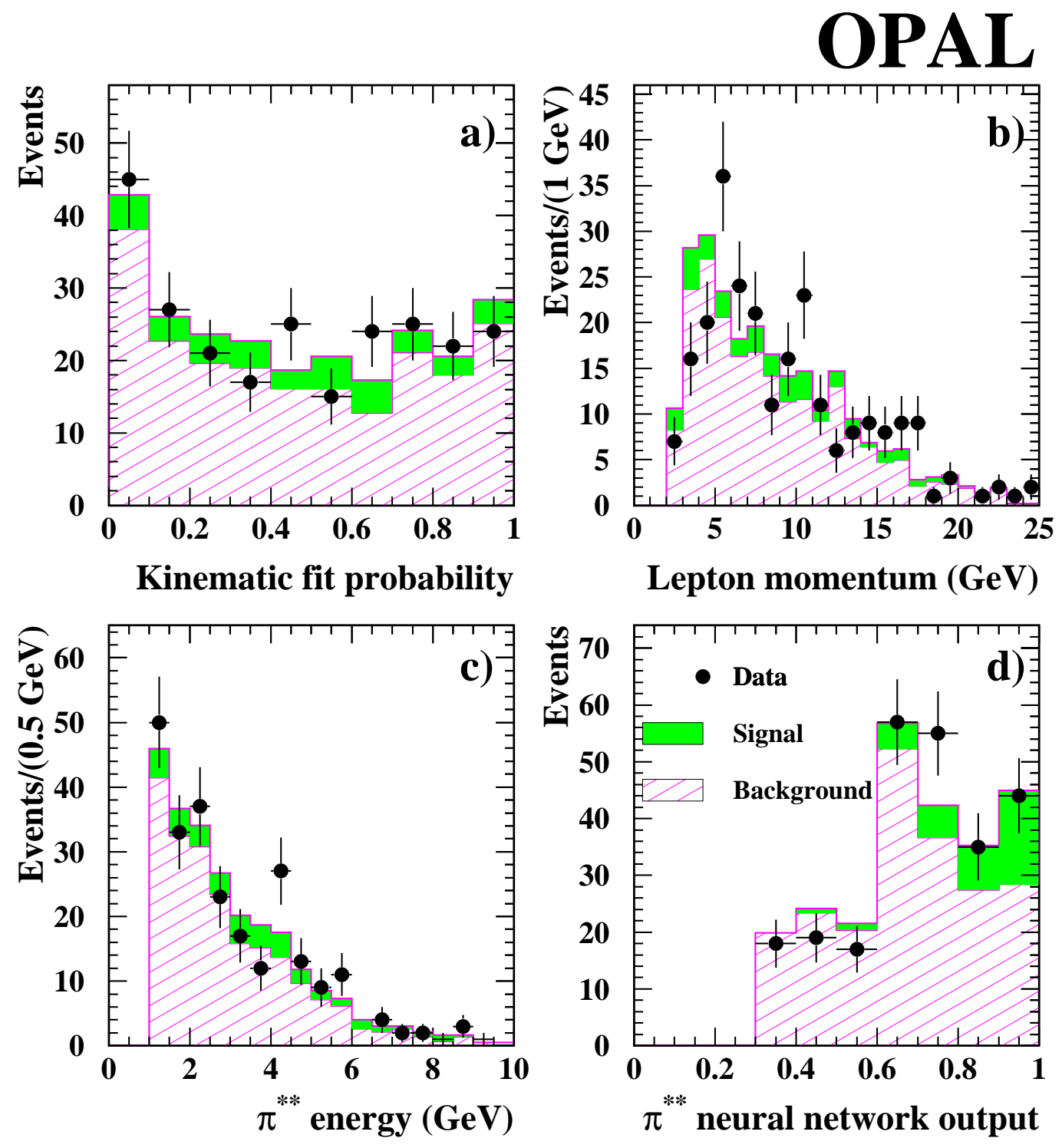

Figure 2: Distributions of data, simulated signal, and backgrounds after all cuts for: a) probability of kinematic fit, b) lepton momentum, c) $\pi^{* *}$ energy, d) $\pi^{* *}$ neural network output. Both $\mathrm{D}^{0}$ decay modes were combined, and the signal rate is the one measured in this paper. 
OPAL
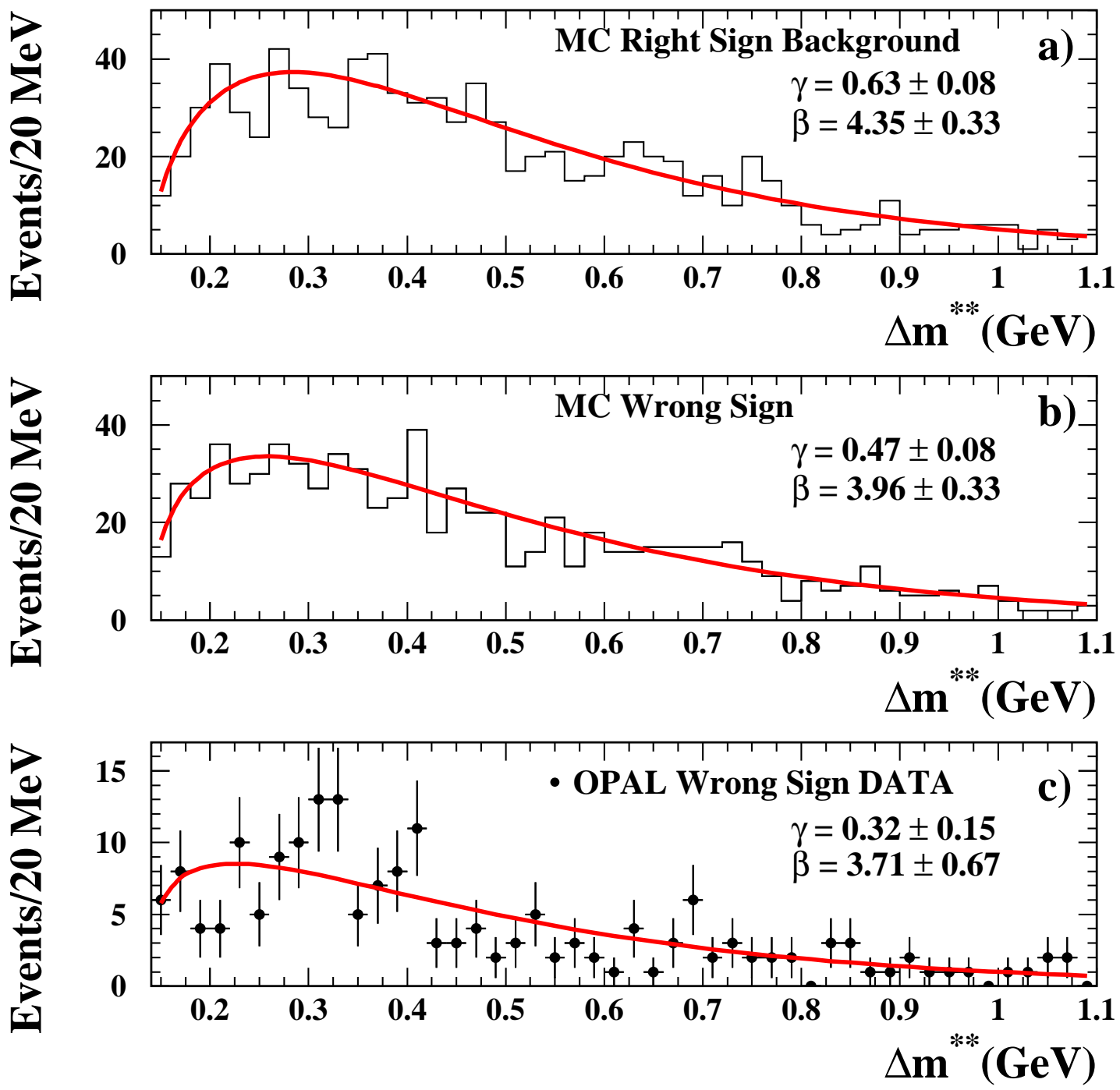

Figure 3: $\Delta m^{* *}$ distributions for the combined modes $\mathrm{D}^{0} \rightarrow(\mathrm{K} \pi$ or $\mathrm{K} 3 \pi)$ : a) Monte Carlo background right sign events, b) Monte Carlo background wrong sign events, c) OPAL wrong sign $\Delta m^{* *}$ data. The superimposed lines are the fits to the functional form $x^{\gamma} e^{-\beta x}$ with $x=$ $\Delta m^{* *}-m_{\pi}$. 

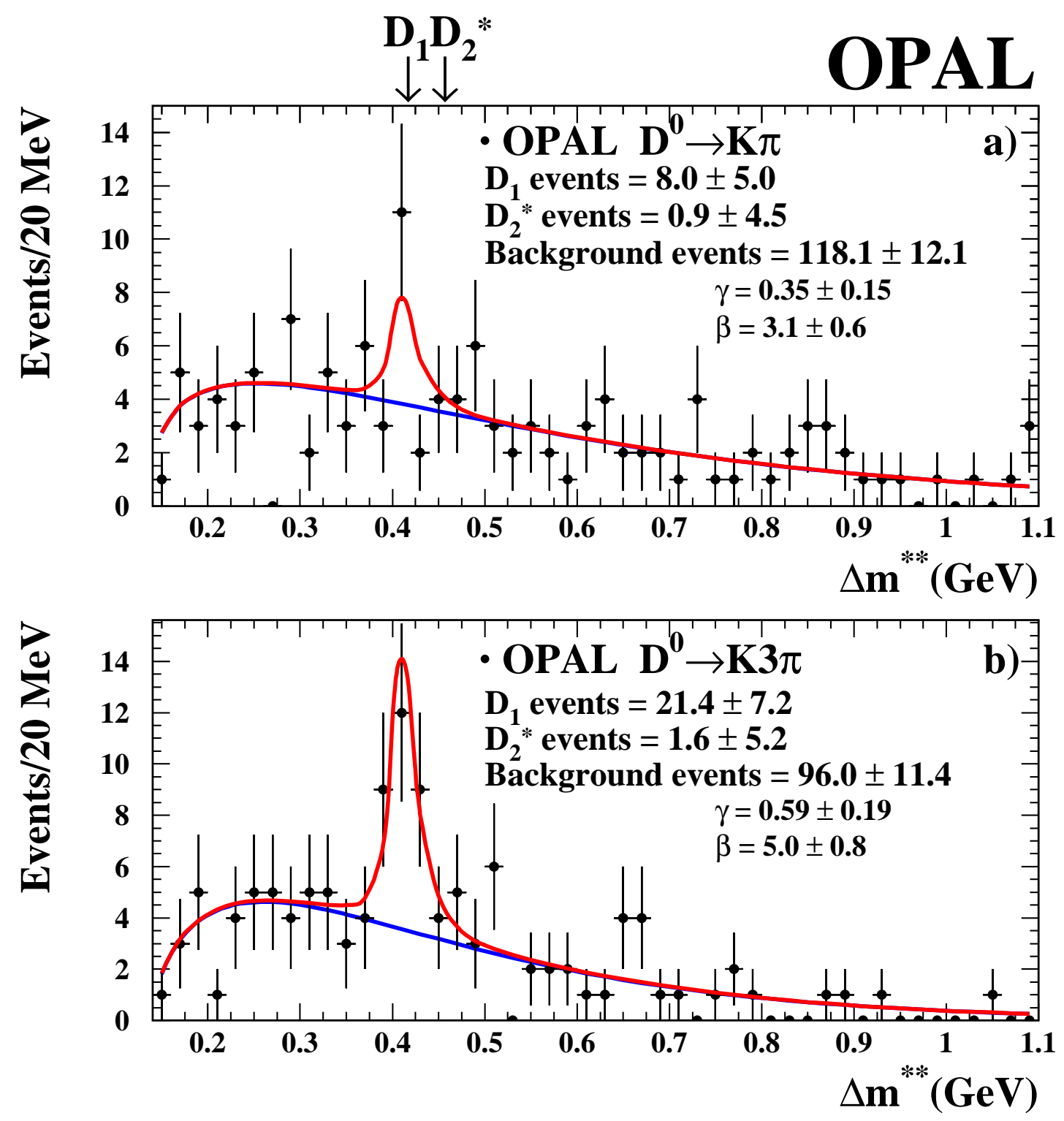

Figure 4: $\Delta m^{* *}$ distribution for a) the $\mathrm{D}^{0} \rightarrow \mathrm{K} \pi$ mode, b) the $\mathrm{D}^{0} \rightarrow \mathrm{K} 3 \pi$ mode. The superimposed lines show the overall fit and the background fitted shape. The expected positions of the $\mathrm{D}_{1}^{0}$ and $\mathrm{D}_{2}^{* 0}$ are indicated by the arrows. 


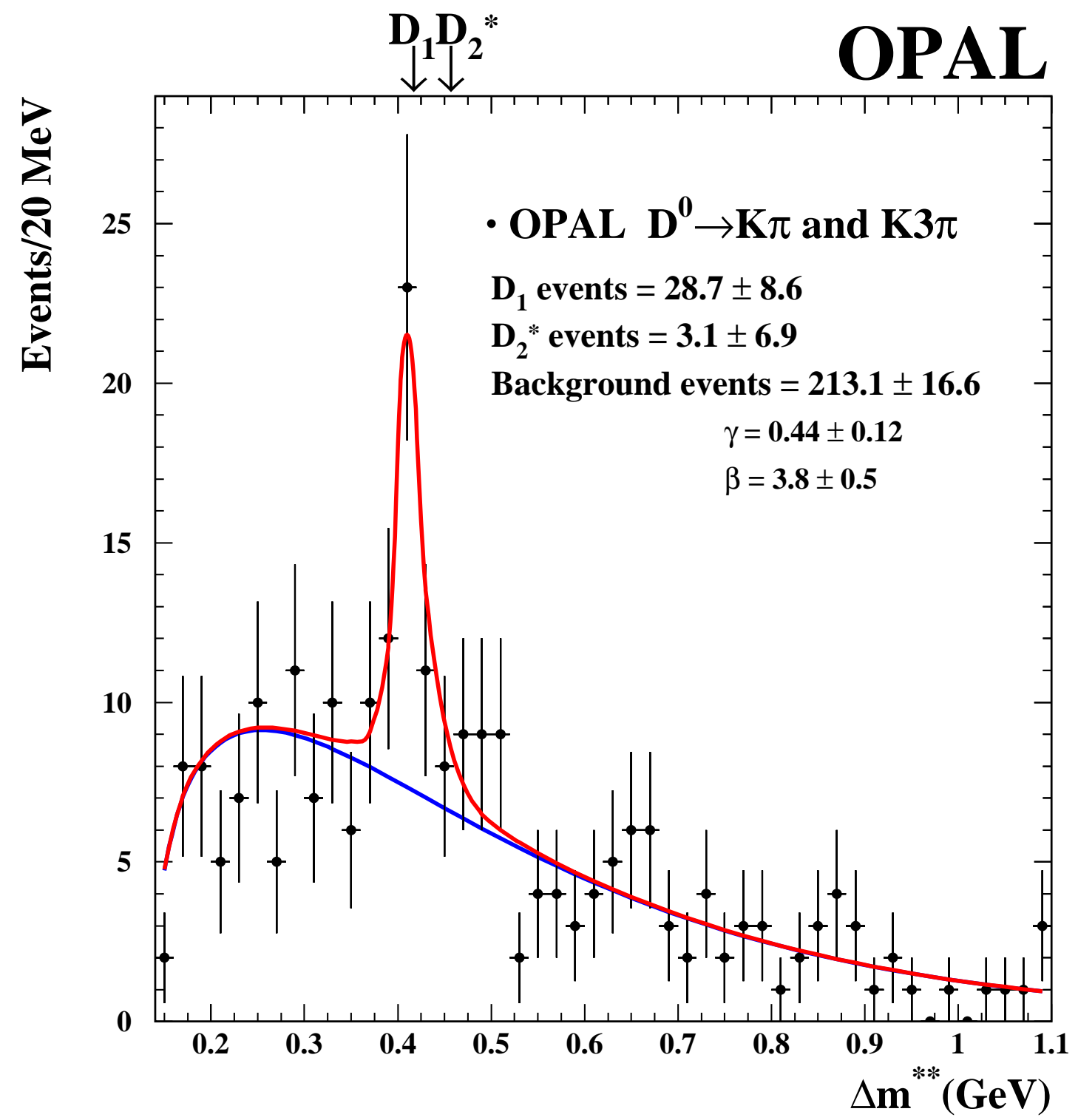

Figure 5: $\Delta m^{* *}$ distribution for $\mathrm{D}^{0} \rightarrow \mathrm{K} \pi$ and $\mathrm{D}^{0} \rightarrow \mathrm{K} 3 \pi$ combined. The superimposed lines show the overall fit and the background fitted shape. The expected positions of the $\mathrm{D}_{1}^{0}$ and $\mathrm{D}_{2}^{* 0}$ are indicated by the arrows. 

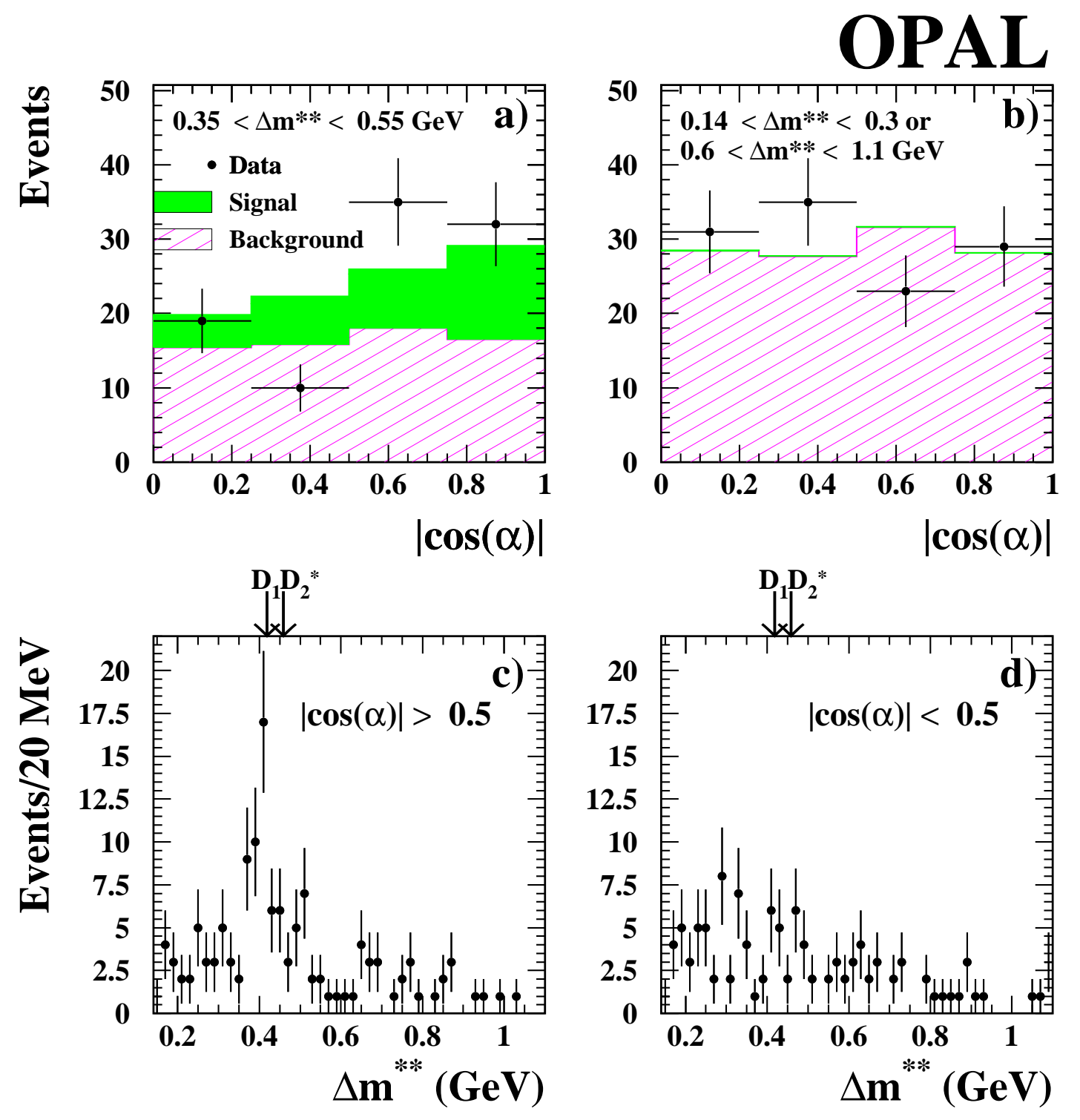

Figure 6: Distributions dependent on the decay angle $\alpha$ : a) $|\cos (\alpha)|$ for the combined $\mathrm{D}^{0} \rightarrow \mathrm{K} \pi$ and $\mathrm{D}^{0} \rightarrow \mathrm{K} 3 \pi$ data and Monte Carlo in the $0.35<\Delta m^{* *}<0.55 \mathrm{GeV}$ signal band; b) complementary events to a) in the $0.14<\Delta m^{* *}<0.3$ and $0.6<\Delta m^{* *}<1.1 \mathrm{GeV}$ sidebands; c) $\Delta m^{* *}$ data distributions for $\mathrm{D}^{0} \rightarrow \mathrm{K} \pi$ and $\mathrm{D}^{0} \rightarrow \mathrm{K} 3 \pi$ combined data in the $|\cos (\alpha)|>0.5$ region (expected to enhance the $\mathrm{D}_{1}$ signal); d) complementary events to c) for $|\cos (\alpha)|<0.5$ (expected to suppress the $\mathrm{D}_{1}$ signal). The expected positions of the $\mathrm{D}_{1}$ and $\mathrm{D}_{2}^{*}$ are indicated by arrows. 